\title{
Hemobilia and Pancreatitis as Complications of a Percutaneous Transhepatic Cholangiogram
}

\author{
Helen C. Redman, MD, and Ramon R. Joseph, MD
}

Percutaneous transhepatic cholangiography (PTC) has become widely accepted as a valuable procedure in differentiating cholestatic jaundice from extrahepatic obstructive jaundice and also in demonstrating the site and nature of the obstruction. Patients are usually operated upon shortly following the procedure if an obstructing lesion is found, decreasing the risk of bile peritonitis and intraperitoneal bleeding, the two most common and most serious complications of PTC (1-3). A Gram-negative septicemia is another serious complication which may result from direct leakage of infected bile into the hepatic venous circulation $(3,4)$. Other uncommon complications including pneumothorax and laceration of the gallbladder have also been reported. We performed a PTC on a 23-year-old clinical-laboratory technician who developed hemobilia, common bile duct obstruction, and clinical pancreatitis following the procedure. He also underwent celiac angiography after the PTC. We report this case because of this unusual complication and because of the picture seen at celiac angiography.

\section{CASE REPORT}

The patient was a 23-year-old male who was hospitalized for hepatitis $B$ antigen-negative hepatitis. The patient was employed as a pathology laboratory technician and gave a history of having accidentally cut himself with a scalpel used at the autopsy of a jaundiced patient three months

From the Departments of Radiology and Internal Medicine, Wayne County General Hospital, Eloise, Michigan, and University of Michigan Medical School, Ann Arbor, Michigan.

Address for reprint requests: Dr. Helen Redman, Mount Zion Hospital and Medical Center, Box 7921, San Francisco, California 94120. prior to the onset of his illness. Initial laboratory studies included a serum glutamic oxaloacetic transaminase level (SGOT) of 1200 units and a serum bilirubin value of 10 $\mathrm{mg} / 100 \mathrm{ml}$. He was treated on an ambulatory basis with satisfactory progress for six weeks when he noted the onset of intense pruritis, increasing jaundice, and light-colored stools. Laboratory studies changed to show a more obstructive picture, with the bilirubin level rising to $45 \mathrm{mg} / 100 \mathrm{ml}$ (50\% direct), SGOT 110 units, and alkaline phosphatase 312 units, rising to 550 units. The stools were never acholic. Prothrombin time was normal. A percutaneous liver biopsy was interpreted as intrahepatic and canalicular cholestasis (Figure 1) and nonspecific portal triaditis (Figure 2). However, with the persistence of the obstructive-type biochemical profile, it was decided to perform a PTC to exclude significant extrahepatic obstruction. Three attempts to enter a bile duct using a Teflon-sheathed 18-gauge needle were made under television fluoroscopic control using both aspiration and injection of contrast material. No duct was entered. The patient tolerated the procedure well and there was no clinical evidence of hemorrhage or bile leakage. The following day celiac angiography was performed to exclude any unsuspected hepatic or biliary abnormality (Figure 3 6). The major hepatic arteries were normal with no displacement or occlusion. In the capillary phase, however, there was a triangular area of hepatic parenchyma that developed a markedly increased stain in the target area of the PTC. Small tortuous draining vessels were seen in this area.

Seven days after the PTC the patient developed severe episodic right upper quadrant pain suggesting acute common duct obstruction. Recurrent episodes of this pain persisted for 25 days. Amylase which had been normal throughout his illness was found to be 360 Somogyi units on the 7 th day, and rose to 975 units after a severe attack of right upper quadrant pain on the 24th day. On the 28th day after PTC, the patient developed melena, but his hematocrit reading did not significantly alter. ${ }^{131} \mathrm{I}$ Rose Bengal liver scan suggested extrahepatic obstruction. Technesium $99 \mathrm{M}$ liver scan suggested diffuse hepatocellular dysfunction.

Celiotomy and common duct exploration 32 days after the PTC revealed blood clots obstructing the common bile duct. These were removed by irrigation. No vascular repair was necessary. T-tube operative cholangiogram was done, and the patient made an uneventful recovery. 


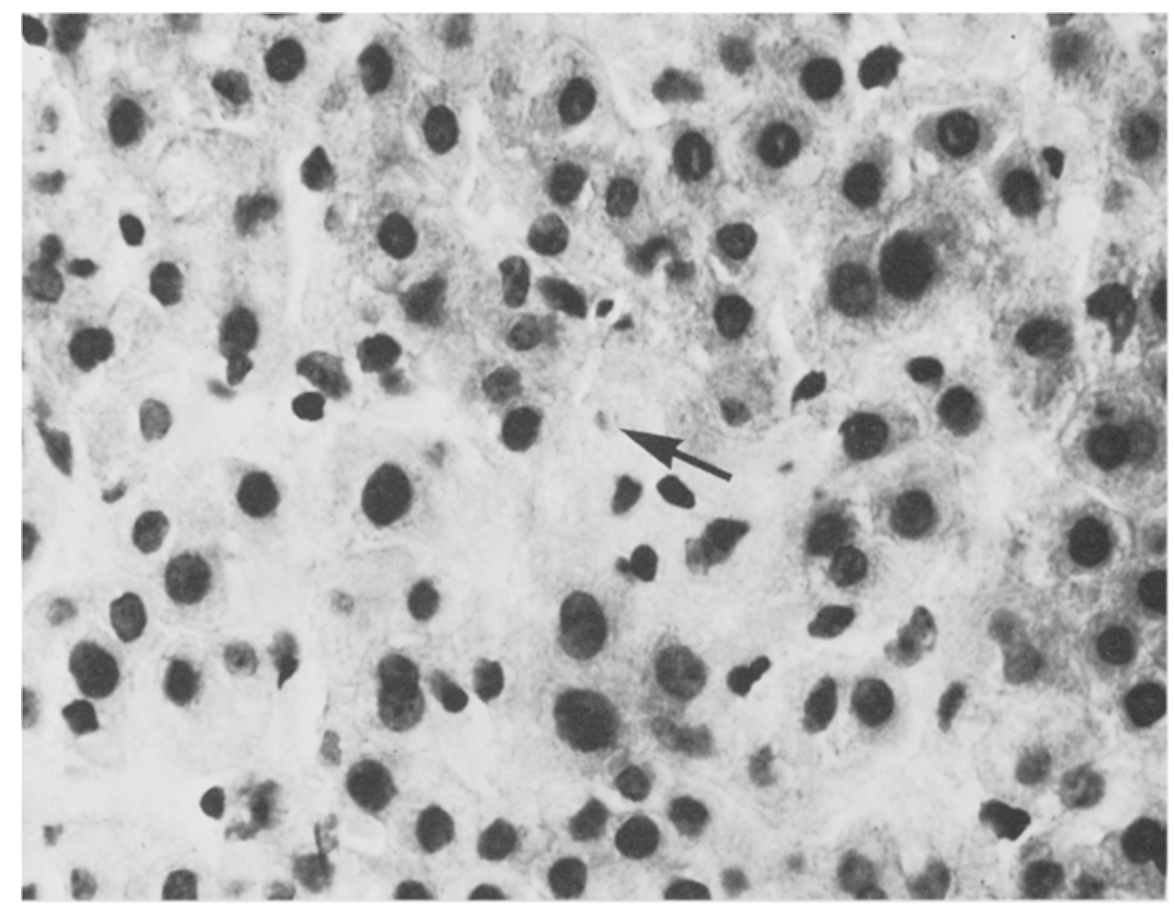

Fig 1. Photomicrograph of percutaneous liver biopsy. Hematoxylin-eosin preparation. Arrow indicates intrahepatic bile plug.

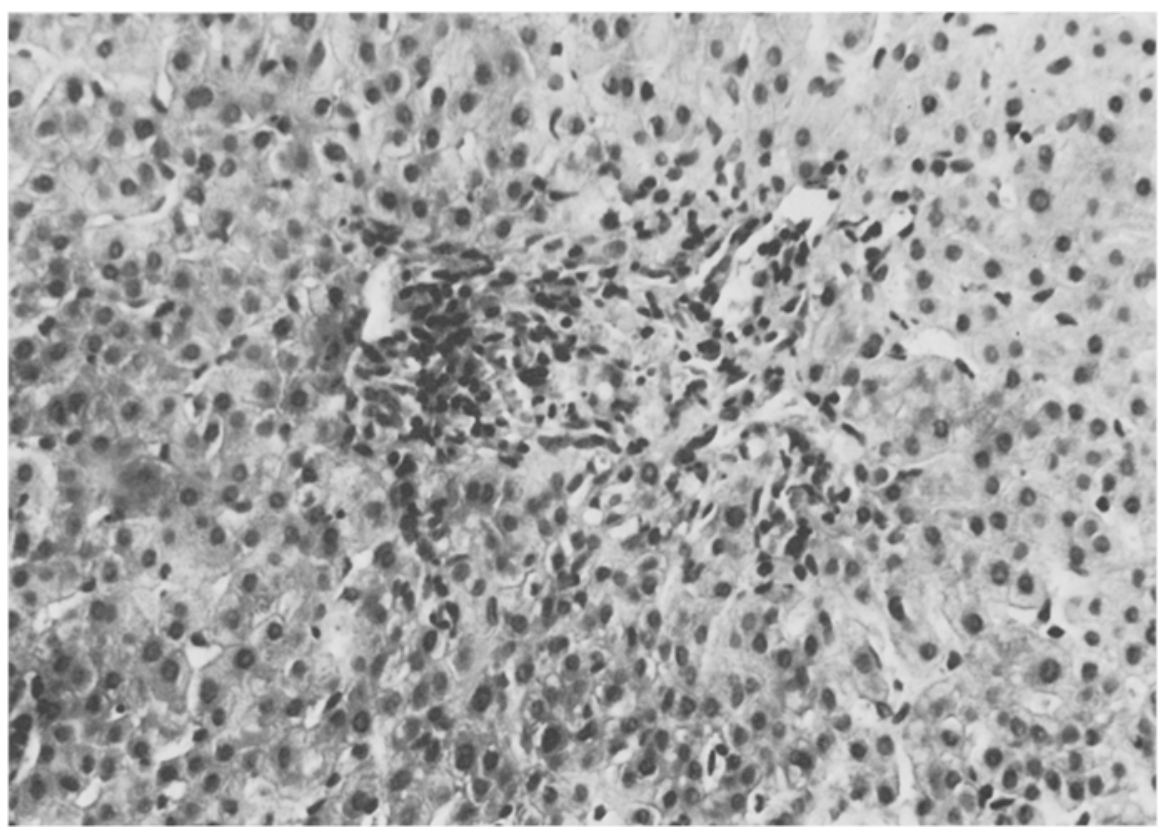

Fig. 2. Photomicrograph of percutaneous liver biopsy. Hematoxylin-eosin stain. Portal area showing lymphocytic infiltration interpreted as a nonspecific portal triaditis. 


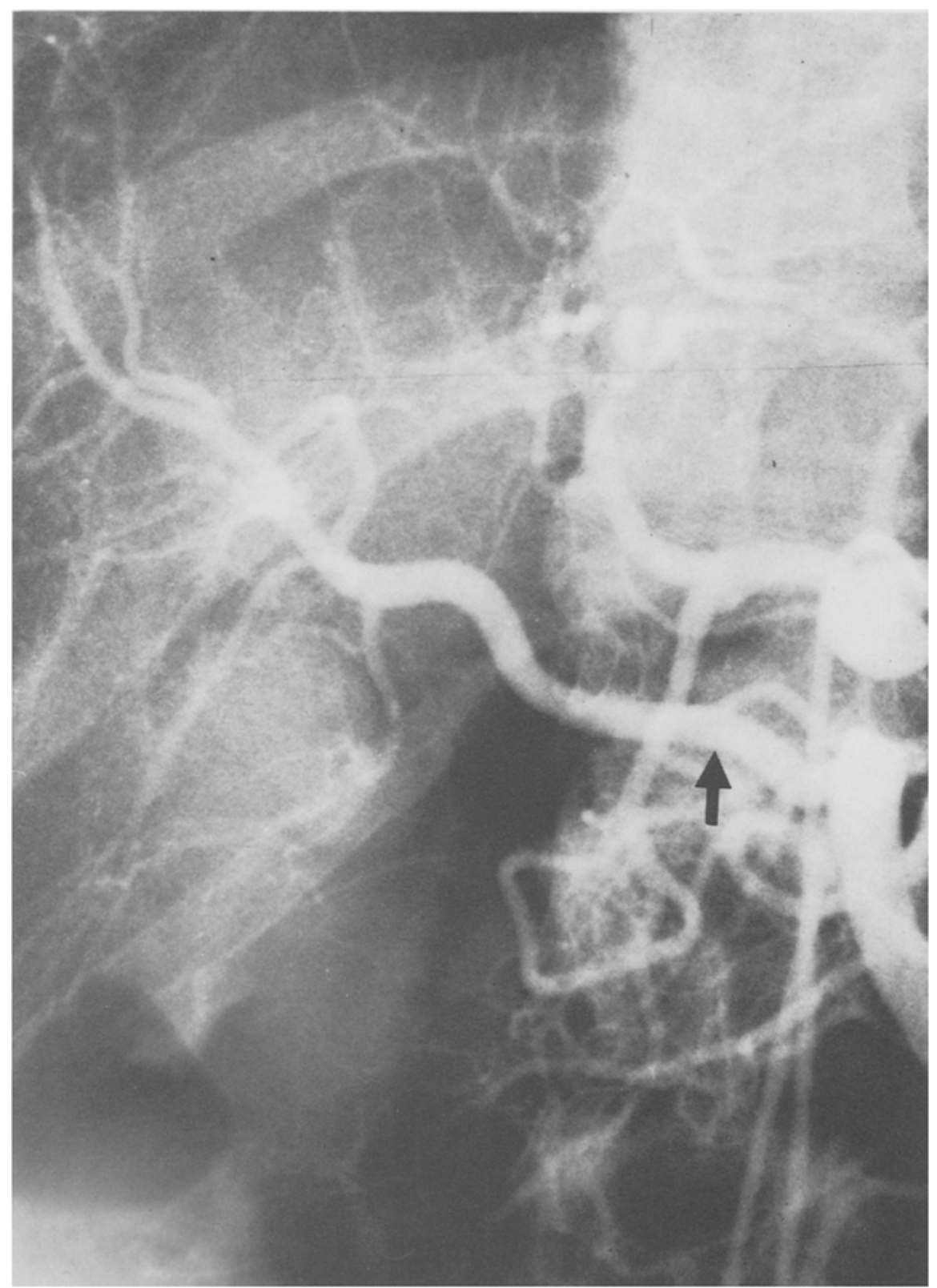

Fig 3. The right hepatic artery has a replaced origin from the superior mesenteric artery. 


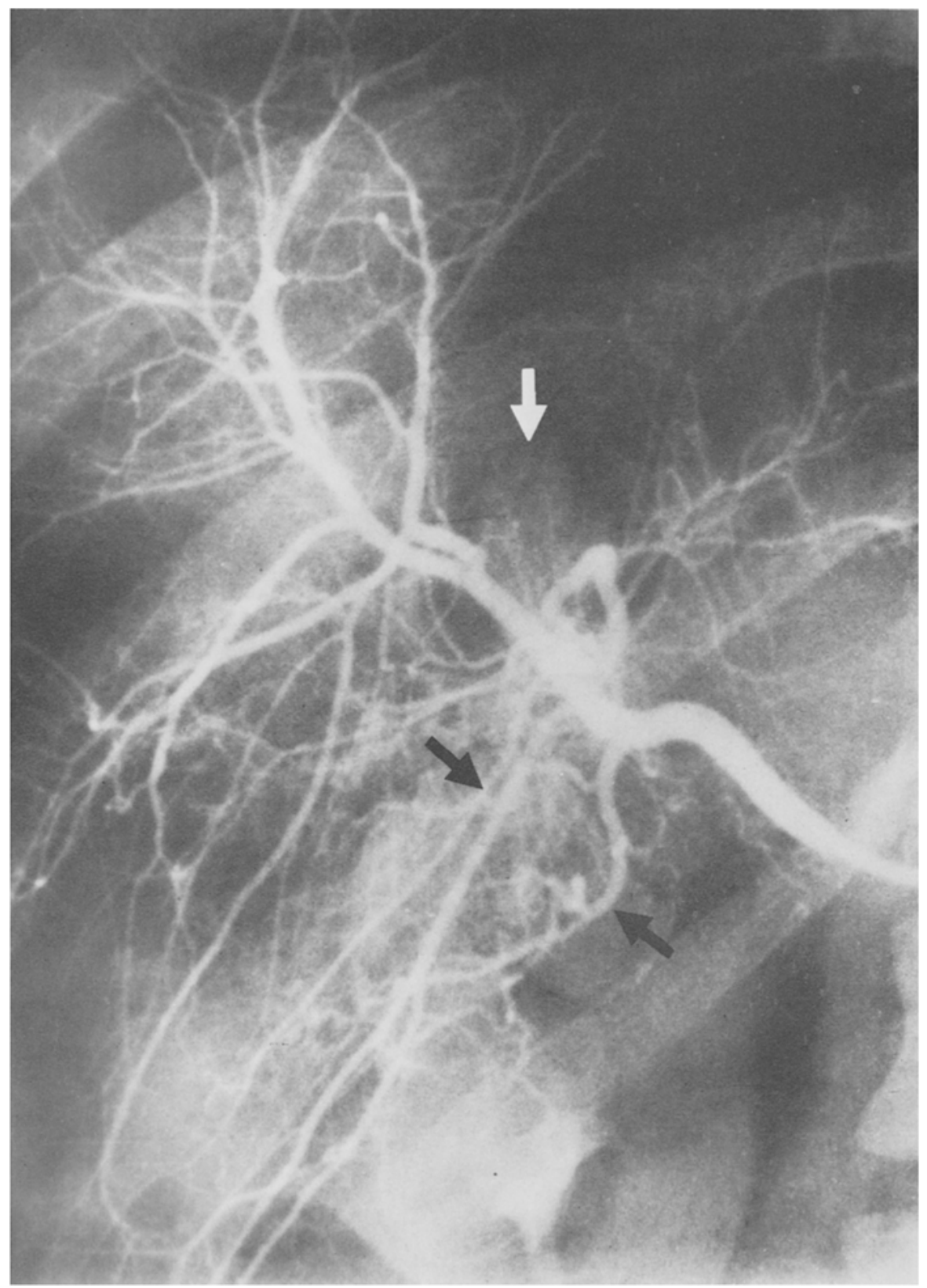

Fig 4. Right hepatic angiogram, arterial phase: The small branches of the deep and superficial cystic arteries (black arrow) are prominent. An early parenchymal liver stain is seen (white arrow). Vascular pattern in the remainder of the right lobe is normal. 


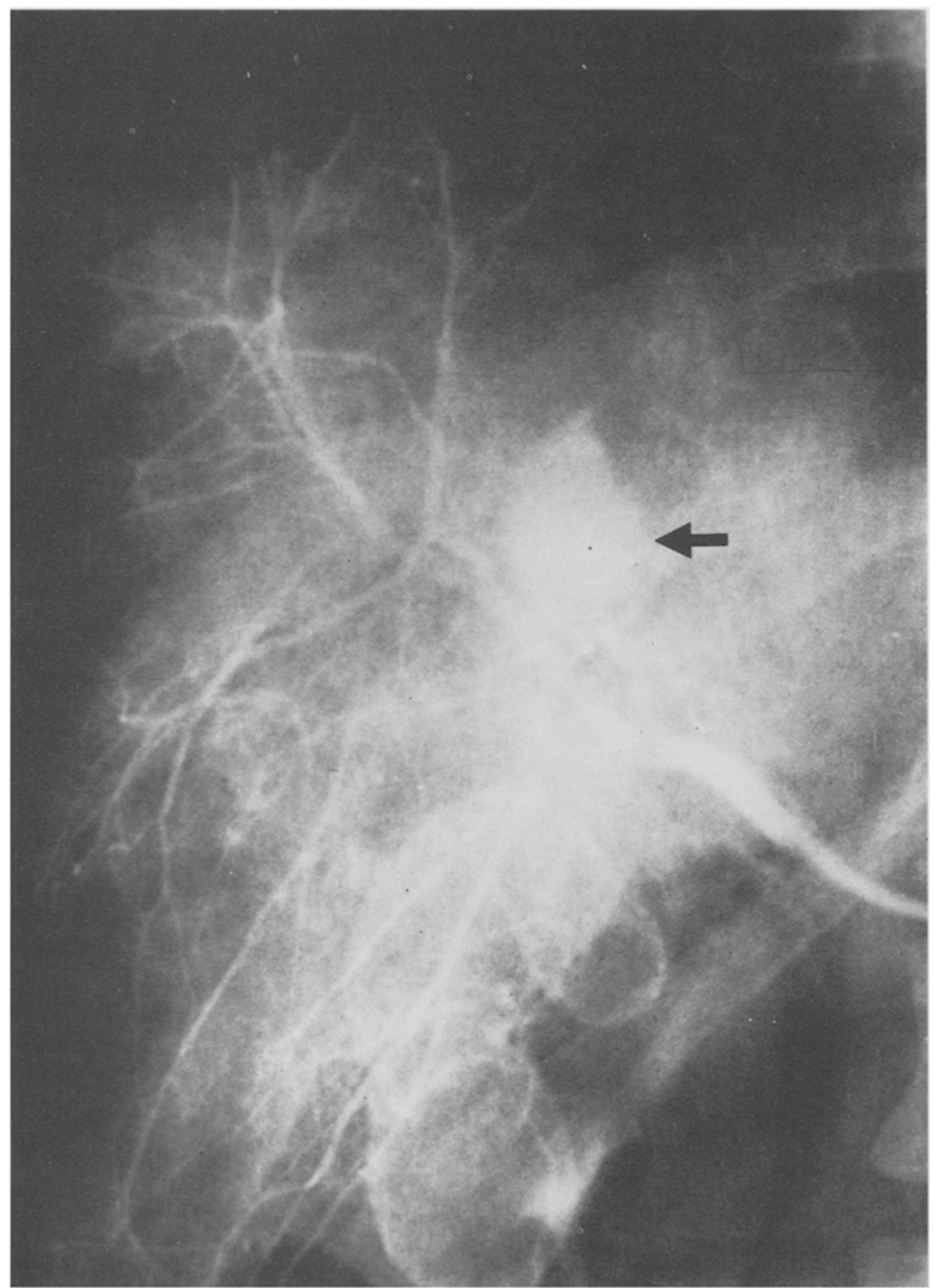

Fig 5. Late arterial phase: A dense parenchymal stain (arrow) has developed in the target area of PTC. The gallbladder wall is well seen. 


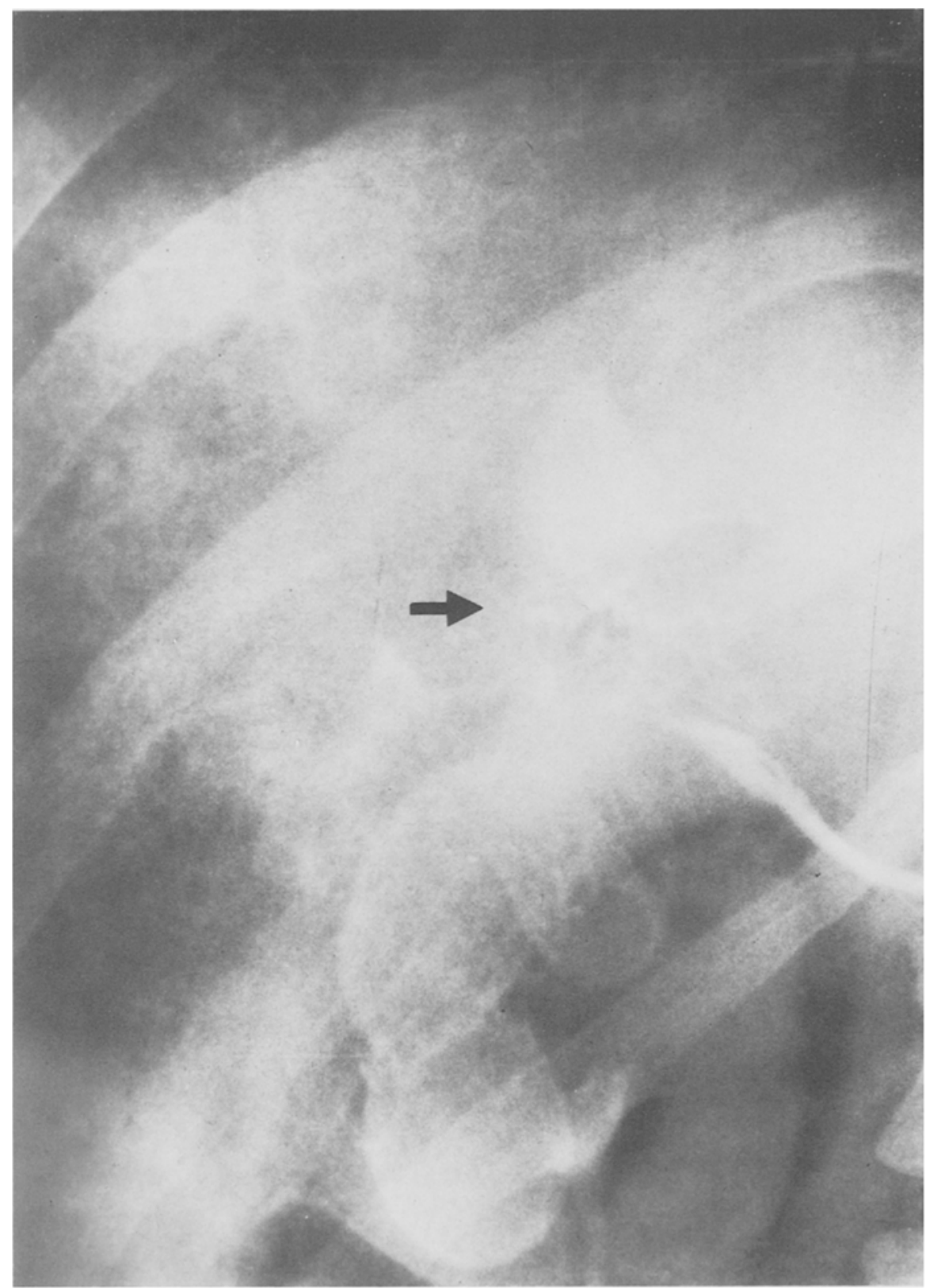

Fig. 6. Late parenchymal phase: Wormian draining vessels (arrow) are seen adjacent to the area of increased parenchymal stain. These channels are similar to those seen in the BuddChiari syndrome. Parenchymal accumulation of contrast medium throughout the remainder of the right lobe was slightly mottled. 


\section{DISCUSSION}

Hemobilia is a rather uncommon clinical problem from any cause. Blunt abdominal trauma is the most frequent cause but hemobilia may be secondary to surgery, especially following liver biopsy and other needling procedures (5). Other less common causes include ruptured hepatic aneurysm, gallbladder disease, hepatic neoplasms, and, in the Far East, ascariasis. If the bleeding is slight, anemia may be the only symptom. When bleeding is more substantial, jaundice, paroxysmal pain, and hemorrhage into the gastrointestinal tract occur. In massive bleeding, hepatic angiography can be useful in the diagnosis and location of the bleeding artery. Clinical awareness of hemobilia is important if the proper diagnosis is to be reached.

Complications following PTC are not common, with a reported frequency of less than $5 \%(1,4)$. Bile leakage is almost universal at laparotomy following PTC in an obstructed biliary system though the volume is frequently small and significant morbidity slight. Some bleeding is also common but is seldom of significance. Gram-negative septicemia may occur if the obstructed biliary system is infected.

Hemobilia is also known to occur during PTC $(3,5)$. Clots in a distended common duct can simulate gallstones or obscure a distal obstructing lesion. The complication occurring in this patient is unusual in that no bile duct was demonstrated during the PTC, although small ducts undoubtedly were entered and in that the complication developed days after the procedure. While hemobilia frequently has a delayed onset after trauma, complications of PTC are generally expected to occur during the procedure or within the next 24-48 hours. Complications are also unusual in nonobstructed patients. The complication was serious, eventuafly necessitating surgical intervention. The findings at celiac angiography were considered to be secondary to the trauma of the PTC. The area of staining was in the target area of all three PTC punctures. The draining vessels were different from normal hepatic veins and it was felt that they might be lymphatics. Their resemblance to the abnormal vascular channels seen in the Budd-Chiari syndrome is remarkable. Possibly an hepatic venous branch was occluded during the PTC and the same vascular channels were draining the liver segment. Clain, however, states that the vessels seen in the Budd-Chiari syndrome are not hepatic lymphatic channels (6).

\section{CONCLUSION}

PTC is a simple and useful tool for differentiating extrahepatic obstructive jaundice from cholestatic jaundice. It demonstrates the level of the obstruction and may determine the nature of the lesion, aiding in the surgical approach. However, it does carry significant risks.

\section{SUMMARY}

Percutaneous transhepatic cholangiography (PTC) was performed on a 23-year-old male because of an atypical progression of hepatitis B antigen-negative hepatitis. No bile duct was entered and the procedure was uneventful. However, celiac angiography the day following PTC revealed abnormal liver vessels in the target area and the patient developed hemobilia and clinical pancreatitis, causing common duct obstruction. Symptomatology persisted until celiotomy 32 days after PTC. Clots were found obstructing the common bile duct.

This case is presented both because of the unusual complications of PTC and the unusual angiographic abnormalities. It is suggested that PTC be reserved for the evaluation of patients when there is a specific indication for the procedure either to differentiate cholestatic jaundice from extrahepatic jaundice or to localize a site of obstruction before surgical intervention. 


\section{REFERENCES}

1. Hines C Jr, Ferrante WA, Davis WD Jr, Tutton RA: Percutaneous transhepatic cholangiography. Am J Dig Dis 17:868-874, 1972

2. Kittredge RD, Finby N: Percutaneous transhepatic cholangiography. Am J Roentgenol Radium Ther Nucl Med 101:592-604, 1967

3. Morettin LB, Dodd GS: Percutaneous transhepatic cholangiography, Am J Dig Dis 17:831845,1972
4. Fleming MP, Carlson HC, Adson MC: Percutaneous transhepatic cholangiography: The differential diagnosis of bile duct pathology. Am J Roentgenol Radium Ther Nucl Med 116:327336, 1972

5. Bismuth H: Hemobilia. N Engl J Med 288:617619,1973

6. Clain D, Freston J, Krell L, Sherlock S: Clinical diagnosis of the Budd-Chiari syndrome. Am J Med 43:544-554, 1967 